\title{
Calidad de vida como respuesta al tratamiento intravesical con toxina botulínica tipo $A$, en el síndrome de vejiga hiperactiva refractaria a tratamiento convencional
}

\author{
Rosa Lucía Álvarez M. ${ }^{1}$, Rocío González S. ${ }^{1}$, Víctor Manuel Elizalde V. ${ }^{2}$ \\ ${ }^{1}$ Departamento de Uroginecología del Hospital de Ginecología y Obstetricia del Instituto Materno Infantil del Estado de \\ México. ${ }^{2}$ Universidad Autónoma del Estado de México, México.
}

\section{RESUMEN}

Objetivo: Determinar la calidad de vida relacionada con salud (CVRS) en mujeres con vejiga hiperactiva (VH) posterior a tratamiento con toxina botulínica tipo A. Método: Se realizó análisis de casos de pacientes con VH utilizando versión española del "King's Health Questionnaire" (KHQ). Resultados: Se estudiaron 10 casos con diagnóstico de $\mathrm{VH}$, el síntoma predominante fue la incontinencia urinaria de urgencia en $70 \%$ de los casos. De acuerdo a los puntos del KHQ se observó mejoría con la aplicación de toxina botulínica de 53,4 a 11,4 puntos post aplicación. Los efectos secundarios fueron retención urinaria (1 caso) y fiebre (1 caso). Presentando recaída el $2 \%$, aplicando nueva dosis de toxina botulínica. Conclusiones: El tratamiento con toxina botulínica intravesical es efectivo para el síndrome de $\mathrm{VH}$ refractaria.

\section{PALABRAS CLAVE: Vejiga hiperactiva, toxina botulínica, calidad de vida relacionada con salud}

\section{SUMMARY}

Objective: To determine the health-related quality of life (HRQol) in women with overactive bladder (OB) syndrome after treatment with botulinum toxin. Method: It realize a cases analysis study of patients with OB syndrome used Spanish version of the "King's Health Questionnaire" (KHQ). Results: The study was realized in 10 cases of patients with $\mathrm{OB}$ syndrome. The predominant symptom was urge incontinence in $70 \%$ of cases. According to the points of the KHQ it was observed that the quality of life improves with the intravesical application of botulinum toxin from 53.4 to 11.4 points after application of treatment. Among the side effects were fever ( 1 case) and urinary retention ( 1 case). Featured relapse $2 \%$ of patients which required a new dose intravesical botulinum toxin. Conclusions: Medical treatment with intravesical botulinum toxin constitutes an effective treatment for refractory OB syndrome.

\section{KEY WORDS: Overactive bladder, botulinum toxin, health-related quality of life}

\section{INTRODUCCIÓN}

La cuarta Consulta Internacional sobre Incontinencia (1) y la International Continence Society, definen a la vejiga hiperactiva $(\mathrm{VH})$ como un síndrome caracterizado por urgencia miccional asociada a frecuencia y nicturia con o sin incontinencia de urgencia $(1,2)$, cuyos sinónimos son: síndrome de urgencia o síndrome de urge-frecuencia $(2,3)$.

La incidencia anual de incontinencia urinaria (IU) en mujeres es del 2 al $11 \%$. La incidencia de vejiga hiperactiva (VH) ocurre entre el 4 y $6 \%$. La IU afecta 
del 5 al $69 \%$ de mujeres (1). La IU de urgencia y la mixta son responsables del $51 \%$ de los casos, $33 \%$ de pacientes con VH tiene IU de urgencia (3).

En México, un estudio reportó al $23 \%$ de mujeres mayores de 30 años, con diagnóstico de $\mathrm{VH}$ (4). El impacto económico y clínico del síndrome no se limita a la enfermedad, también implica mayores costos médicos asociados con enfermedades concomitantes $(3,5)$.

Existen factores de riesgo como: enfermedades infecciosas, menopausia y climaterio, prolapso genital y diversas alteraciones urinarias, neoplasias ginecológicas, trastornos neurológicos, diabetes mellitus $(4,6)$, obesidad, sedentarismo, estrés, etc. $(4,5,7)$. La etiología es idiopática en la mayoría de los casos (1). La triada clásica incluye urgencia miccional, nicturia y aumento en la frecuencia urinaria $(8,9)$. El diagnóstico incluye la escala de calidad de vida, la cistoscopia y el estudio urodinámico. El tratamiento debe considerar cada caso en forma individual y adaptarlo a su realidad.

El tratamiento no quirúrgico es el pilar del tratamiento para la $\mathrm{VH}$. Se deben considerar procedimientos quirúrgicos, especialmente en casos refractarios al manejo establecido $(1,10,11-13)$. La toxina botulínica Tipo A es un tratamiento alternativo $(7,14-19)$.

El objetivo de esta comunicación es determinar la calidad de vida en mujeres con vejiga hiperactiva posterior a tratamiento intravesical con toxina botulínica refractaria a tratamiento convencional.

\section{PACIENTES Y MÉTODO}

Se realizó un análisis de casos en 10 pacientes que acudieron al servicio de urología ginecológica del Hospital de Ginecología y Obstetricia del Instituto Materno Infantil del Estado de México, México, con diagnóstico de síndrome de $\mathrm{VH}$ refractaria a tratamiento convencional. A todas se les realizó una evaluación urológica consistente en anamnesis, examen físico, y diario miccional por 3 días. Se les aplicó el cuestionario de salud King's Health Questionnaire que mide el impacto de la incontinencia urinaria sobre la calidad de vida relacionada con salud (CVRS) e incorpora la evaluación objetiva de síntomas urinarios. Es un cuestionario autoadministrado, se interpreta: 0 a 30 puntos como incontinencia urinaria leve, 33 a 47 puntos como incontinencia urinaria moderada, y 47 a 64 puntos como incontinencia urinaria severa $(6,7,20-22)$.

A todas las pacientes se les realizó: examen general de orina, urocultivo, pruebas de función renal, ecografía renal y vesical, cistometría, cistoscopia y estudio de urodinamia monocanal con medición de actividad bioeléctrica de piso perineal. La exploración física sistemática incluyó: exploración abdominal, pélvica y perineal. Se realizó prueba de Valsalva para detectar pérdidas secundarias a in- competencia esfinteriana. Se evalúo la presencia de prolapso de órganos pélvicos o atrofia genitourinaria y la función voluntaria de los músculos del suelo de la pelvis mediante exploración vaginal o rectal.

La medicación anticolinérgica se interrumpió 2 semanas antes de la evaluación urodinámica. Se realizó el estudio urodinámico 30 días antes de la aplicación de toxina botulínica tipo A (TBTA). En posición de litotomía, bajo neuroleptoanalgesia y mediante el uso de aguja Prostaject bajo visión directa con cistouretroscopio rígido con telescopio de 30 grados, camisa de $21 \mathrm{fr}$, con puente de doble vía, cámara de video y fuente de luz marca Olimpus, se realizó la inyección de 300 Unidades de TBTA (Botox®) diluidos en $30 \mathrm{ml}$ de solución fisiológica divididos en 30 sitios de punción de $1 \mathrm{ml}$ cada una repartidas en todas las caras de la vejiga, respetando el trígono para evitar posibilidad de reflujo vesicoureteral. El seguimiento clínico se realizó a través del diario miccional a la semana 1 y 4 , y meses $3,6,12$ y 24, con la finalidad de evaluar una segunda aplicación de toxina botulínica si así lo ameritara la paciente. Se aplicó cuestionario de salud King's a las 4 semanas pos aplicación de TBTA intravesical. Para el análisis estadístico se utilizó la prueba $t$ de Student para muestras relacionadas, con una significancia estadística de $p<0,05$.

Se solicitó consentimiento bajo información y autorización quirúrgica a las pacientes, se mantuvo la confidencialidad y el anonimato de la información recolectada. Se cumplieron las reglas de la ley General de Salud del Estado de México y la Declaración de Helsinki, sobre investigación en humanos. El estudio fue aprobado por el Comité de Ética Institucional.

\section{RESULTADOS}

Se estudiaron 10 pacientes con diagnóstico de síndrome de $\mathrm{VH}$ refractaria a tratamiento. El rango de edad del grupo estudio fue de 40 a 71 años, con una media de 54,3 $\pm 11,8$ años, moda de 54 años. Todas las pacientes se dedicaban al hogar, estaban unidas legalmente, $90 \%$ eran multigestas, con una media de 5 embarazos. Con respecto a enfermedades concomitantes, $30 \%$ no portaban enfermedad crónica, $40 \%$ presentaban diabetes mellitus tipo 2 bajo control metabólico, $20 \%$ hipotiroidismo y $10 \%$ lupus eritematoso sistémico.

Respecto al diario miccional se observó que $10 \%$ cursaban con urgencia urinaria, 20\% aumento en la frecuencia de micciones, nictámero (veces que el paciente orina en 24 horas) mínimo fue de 14 y máximo de 16 veces, $70 \%$ cursaban con incontinencia urinaria de urgencia al momento del diagnóstico por reporte del diario miccional, $10 \%$ con urgencia urinaria y $20 \%$ con aumento de la frecuencia urinaria. 
El $100 \%$ de las pacientes tuvieron "prueba de la toalla" negativa para incontinencia urinaria de esfuerzo. Durante la cistometría se observó que el primer deseo se presentó con un mínimo de $30 \mathrm{ml}$, máximo de $156 \mathrm{ml}$, media de $82,5 \mathrm{ml}$. El segundo deseo se presentó con un mínimo de $75 \mathrm{ml}$, máximo de $250 \mathrm{ml}$, y media de $138 \mathrm{ml}$. El deseo imperioso con mínima de $95 \mathrm{ml}$, máxima de $495 \mathrm{ml}$ y media de $248,9 \mathrm{ml}$, observándose una disminución en la capacidad vesical.

El $90 \%$ de las pacientes presentaron contracciones no inhibidas del detrusor y no las presentaba el $10 \%$. La medición de orina residual fue menor a $50 \mathrm{ml}$, con una media de 9,2 $\mathrm{ml}$, mínima de $4 \mathrm{ml}$ y máxima de $24 \mathrm{ml}$, consideradas dentro de parámetros normales (Tabla I).

En relación a resultados del estudio histopatológico: $60 \%$ presentaba cistitis crónica inespecífica, $20 \%$ cistitis crónica folicular y $20 \%$ cistitis crónica intersticial.

Respecto a la evaluación pre y post aplicación de TBTA intravesical se observó que la media de los puntos del King's Health Questionnarie para la evaluación del impacto de la incontinencia urinaria sobre la calidad de vida fue de 53 y 11 puntos respectivamente $(p<0,001)$ (Figura 1$)$.

Tabla I

EVALUACIÓN DE CISTOMETRÍA DE CONSULTORIO

\begin{tabular}{lcccc}
\hline Parámetro evaluado & Frecuencia & $\begin{array}{c}\text { Mínimo } \\
(\mathrm{ml})\end{array}$ & $\begin{array}{c}\text { Máximo } \\
(\mathrm{ml})\end{array}$ & $\begin{array}{c}\text { Media } \\
(\mathrm{ml})\end{array}$ \\
\hline Primer deseo & 10 & 30,0 & 156,0 & 82,5 \\
Segundo deseo & 10 & 75,0 & 250,0 & 138,0 \\
Deseo imperioso & 10 & 95,0 & 495,0 & 248,9 \\
Volumen micción & 10 & 150,0 & 600,0 & 294,1 \\
Orina residual & 10 & 4,0 & 24,0 & 9,2 \\
Capacidad vesical & 10 & 156,0 & 610,0 & 303,3 \\
Diuresis minuto & 10 & 0,60 & 3,9 & 2,02 \\
\hline
\end{tabular}

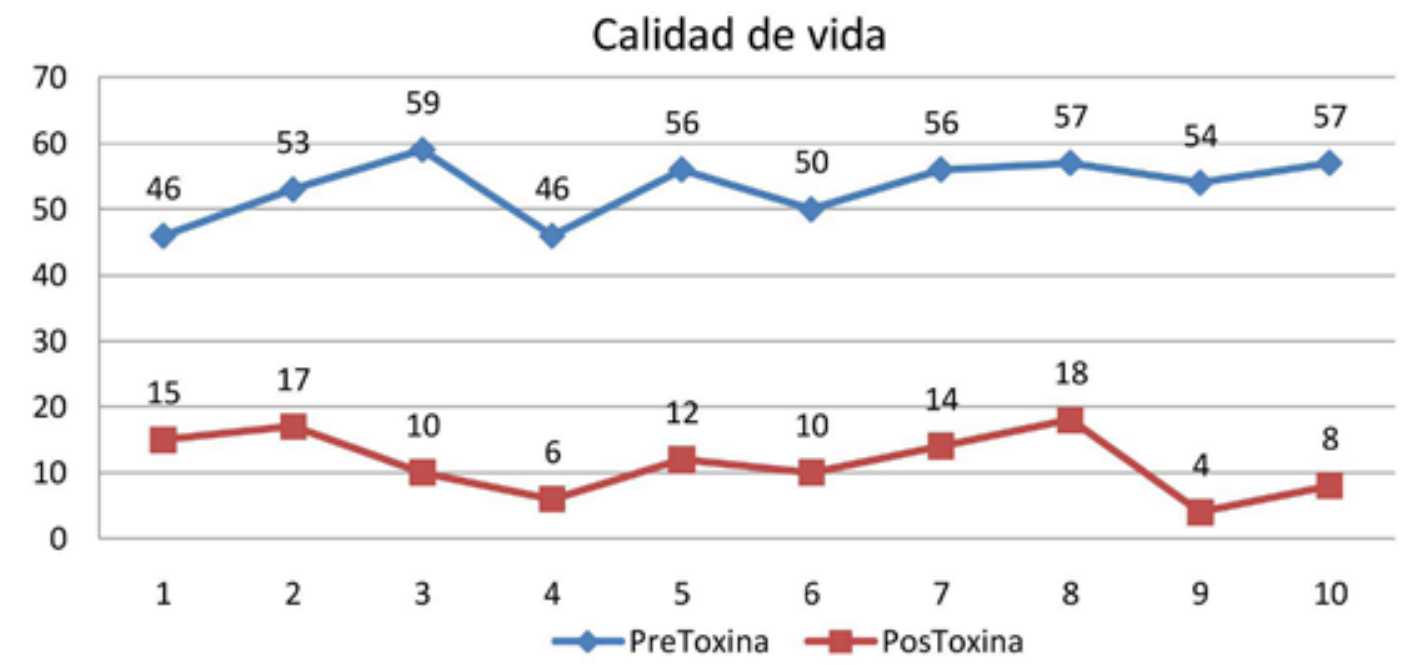

1er Cuestionario de Salud King'spretoxina: 46 a 59 puntos con un promedio de 53 puntos. 2do Cuestionario de Salud King'spostoxina: 4 a 18 puntos con un promedio de 11 puntos. Presentaron una mejoría del $88 \%$ en promedio de su calidad de vida.

Figura 1. Puntuación de calidad de vida según King's Health Questionnaire, antes y después del tratamiento intravesical con toxina botulínica. 
Sólo el $20 \%$ de las pacientes requirieron una segunda aplicación (reinyección) a los 12 y 16 meses, con un incremento de puntaje de 56 y 50 puntos previo a la aplicación, quienes presentaron 10 y 12 puntos respectivamente pos aplicación de TBTA.

Dos pacientes presentaron efectos secundarios pos aplicación de TBTA. Una presentó retención urinaria 48 horas posterior a la aplicación y se trató con sonda con vaciamiento intermitente cada 2 horas, presentando micción voluntaria a las 72 horas. La segunda presentó fiebre de $38^{\circ} \mathrm{C}$ a las 6 horas después de la aplicación TBTA, la cual se trató con 1 gramo de paracetamol en dosis única.

\section{DISCUSIÓN}

Nuestros resultados señalan que de acuerdo a los puntos del KHQ se observó mejoría de 53,4 a 11,4 puntos pos aplicación de TBTA intravesical, en el $100 \%$ de las mujeres con vejiga hiperactiva (VH) refractaria a tratamiento convencional, mejorando significativamente la calidad de vida relacionada con salud (CVRS). Estos resultados son semejantes a los obtenidos por Srikrishna y cols en 2007 (13), quienes informan de mejorías en la calidad de vida y en los síntomas de urgencia, frecuencia e incontinencia. Mientras que Pannek y cols en 2009 (17), reportan que la TBTA se considera una excelente opción terapéutica para el tratamiento de la hiperactividad del detrusor refractaria a tratamiento convencional.

Se ha descrito en la literatura una eficacia del $100 \%$ en más de 600 pacientes tratados por hiperactividad neurogénica del detrusor (OND) y en 200 pacientes con actividad idiopática del detrusor (IDO) y sé informa mejoría en la calidad de vida, en los síntomas de urgencia y frecuencia e incontinencia con mejoras concomitantes en los parámetros urodinámicos (23).

Del Popolo y cols (16) reportan la experiencia con TBTA inyectada dentro del músculo detrusor en pacientes con lesiones de médula espinal en enfermedades que causan incontinencia neurogénica, en el cual se administró 300 unidades de TBTA (Botox®, Allergan Inc.) en repetidas ocasiones y reporta una eficacia estadísticamente significativa $(p<0,001)$ entre la medición inicial y la primera administración, no así con respecto a la administración repetida. No se reportan complicaciones relacionadas con la inyección o efectos secundarios relacionados con la toxina.

A diferencia del anterior, en nuestro estudio se reportan complicaciones como retención urinaria 48 horas post aplicación de inyección de TBTA en una paciente, la cual se manejó con sonda endouretral intermitente con vaciamiento cada dos horas y presentando micción espontanea a las 72 horas posterior al tratamiento. Una segunda paciente presentó fiebre de $38^{\circ} \mathrm{C}$ posterior a la aplicación de inyección de toxina botulínica administrando 1 $\mathrm{g}$ de paracetamol en dosis única. En el presente estudio se efectuó la cistometría de consultorio o mono canal, en donde se observó una mejoría en la capacidad de compliance así como en la presencia o ausencia de contracciones no inhibidas del detrusor. En nuestro estudio el $80 \%$ de las pacientes se mostraron satisfechas con el tratamiento y el $20 \%$ requirió de una segunda aplicación.

Giannantoni y cols (15) en 2009, en su reporte de seis años de seguimiento, cuyo objetivo fue investigar la eficacia y la seguridad de TBTA intradetrusorial en un grupo de pacientes con lesión de médula espinal (SCl) con hiperactividad refractaria del detrusor (DO), refieren una disminución significativa en la frecuencia de episodios de incontinencia diarias $(p<0,01)$, un aumento significativo en el primer deseo de contracción del detrusor no inhibido y en la capacidad máxima de la vejiga $(p<0,001$ para ambos), y una disminución significativa en la presión máxima de estas contracciones $(p<0,01)$. Quince pacientes (82\%) eran completamente continentes, el índice de calidad de vida aumentó significativamente, las limitaciones del estudio se relaciona con el pequeño número de pacientes incluido y concluye que los pacientes con incontinencia urinaria refractaria y no apta para la cirugía invasiva, el tratamiento intravesical con TBTA representa una alternativa válida

Los resultados de nuestro estudio concuerdan con el estudio de Giannantoni y cols (15), sin embargo, nuestros pacientes no tenían lesión de médula espinal, pero si patología de base como la diabetes mellitus (40\%), hipotiroidismo (20\%), lupus eritematoso sistémico (10\%). En nuestro estudio se realizó seguimiento por medio del registro gráfico de micción donde se reportó el $10 \%$ de pacientes con urgencia urinaria y un $20 \%$ de aumento en la frecuencia urinaria, el $100 \%$ de nuestras pacientes no presentaron incontinencia urinaria de esfuerzo por la "prueba de la toalla negativa". Se aplicó el cuestionario de calidad de vida King's Health Questionnaire obteniendo una significancia estadística de $p<0,001$, al igual que el estudio anterior nuestra limitación fue el número de pacientes estudiados.

Las pacientes de este estudio presentan una edad similar a lo reportado por Tyagi y cols en 2006 (3). Respecto a los antecedentes personales patológicos presentes en este estudio se observa una gran similitud en comparación con lo reportado por de Boer y cols (5), quienes en 2011, mencionan que las enfermedades asociadas al síndrome de VH son: enfermedades infecciosas, menopausia y climaterio, prolapso genital e incontinencia urinaria, neoplasias ginecológicas, neoplasias urológicas litiasis urinaria, obstrucción urinaria baja, trastornos neurológicos, diabetes mellitus, obesidad, sedentarismo, estrés, 
tabaquismo, alcoholismo, mujeres de raza blanca, hispánicas y asiáticas, también similar a otros autores $(1,3,4,24)$.

Es de tomar en cuenta que esta patología tiene un impacto económico y clínico que no se limita a la misma enfermedad ya que genera mayores costos médicos asociados con enfermedades concomitantes $(3,5,10,21)$.

El presente estudio no presenta diferencia con respecto a lo reportado por Thüroff y cols en 2011 (1), con respecto a que las manifestaciones clínicas del síndrome de VH son: frecuencia, urgencia, nicturia e incontinencia urinaria, se pueden presentar otras alteraciones como disfunción sexual, depresión, infecciones urinarias, incremento del riesgo de caídas y consecuentemente riesgo de fracturas, pérdida de sueño, miedo, ansiedad, entre otros, por lo que de Boer y cols (5) y Tyagi y cols (3) comentan que lo anterior obliga al paciente a modificar su actividad laboral y social con micciones más frecuentes, restricción en consumo de líquidos, realizar actividades cerca de un baño o bien identificar la localización del mismo, vestir ropa oscura o inclusive toallas sanitarias o pañal.

De los estudios realizados en esta población se observó que la "prueba de la toalla" fue negativa en el $100 \%$ de los casos, la cistometría demostró una reducción en la capacidad vesical con una mínima de $156 \mathrm{ml}$, una máxima de 610 con promedio de $303 \mathrm{ml}$, coincidiendo con lo reportado por Palma y cols (6), quienes en 2010, refieren se debe a un detrusor hiperactivo. En cuanto a la presencia de contracciones no inhibidas del detrusor la cual se presentó en el $90 \%$ de los casos en el presente estudio. Palma y cols (6), encontraron que el $50 \%$ de las mujeres con síntomas de $\mathrm{VH}$ no muestran hiperactividad del detrusor. Yoshida y cols en 2010 (24), refieren que las mujeres con aumento en la sensación de llenado de la vejiga tendrán mayor prevalencia de hiperactividad del detrusor.

La prueba urodinámica proporciona información importante para la administración de tratamiento, seleccionando a los pacientes de riesgo de fracaso al tratamiento. Esta idea va ganando fuerza a medida que surgen nuevos tratamientos quirúrgicos para el síndrome de vejiga hiperactiva, como las inyecciones de toxina botulínica y neuromodulación $(2,24)$. En lo relacionado a la orina residual Palma y cols (6), mencionan que se pueden obtener residuos pos miccionales no confiables debido al exceso de hidratación y consecuente con la distensión vesical exagerada, que resulta en un volumen residual alto y artificial, y muchas veces, residuos bajos falsos, porque son obtenidos después de dos o hasta tres micciones, generalmente no informadas, aunque se ha reportado que el volumen residual esta incrementado en el síndrome de $\mathrm{VH}$ y que el residuo es expresado en unidad de volumen y generalmente no debe ser mayor a 100 a $150 \mathrm{ml}$ (2); lo que coincide con los resultados de este estudio dado que la orina residual presenta una mínima de $4 \mathrm{ml}$, con máxima de $24 \mathrm{ml}$ y media de $9,3 \mathrm{ml}$.

Los estudios histopatológicos coinciden con Yoshida y cols (24), quienes reportan que la disfunción urotelial puede aumentar la cantidad de sustancias liberadas por el urotelio, y conducir a cambios en las propiedades de las vías aferentes de la vejiga, resultando en aumento de los síntomas de la vejiga hiperactiva $(19,24)$

Las limitaciones de nuestro estudio es que la muestra es pequeña dado que se subdiagnostica el problema, lo cual evita que las pacientes lleguen a la Clínica de Uroginecología para su diagnóstico y tratamiento adecuado. Otra limitación es el costo de la TBTA, que es muy elevado y las pacientes atendidas en la institución provienen de estratos socioeconómicos bajos.

De acuerdo a nuestros resultados preliminares se aconseja: la aplicación de TBTA intravesical en las pacientes con síndrome de vejiga hiperactiva refractarias a tratamiento convencional, y la aplicación de cuestionarios de calidad de vida en las pacientes con sospecha de síndrome de vejiga hiperactiva de forma intencionada en la consulta de primer contacto.

\section{CONCLUSIONES}

Hubo un cambio significativo en la calidad de vida en mujeres con $\mathrm{VH}$ refractaria a tratamiento convencional con la aplicación intravesical de TBTA. La causa principal del deterioro en la calidad de vida de las pacientes con síndrome de $\mathrm{VH}$, es la urgencia urinaria seguida del aumento en la frecuencia de micciones con incontinencia urinaria de urgencia, lo que les impide llevar una vida socialmente aceptable.

\section{REFERENCIAS}

1. Thüroff JW, Abrams $P$, Artibani $W$, et al. Guías EAU sobre incontinencia urinaria. Actas Urol Esp 2011;35(7):373-8.

2. Palma P. Urofisioterapia. Caracas, $1^{\text {a }}$ edición. Palma $P$ (ed), 2010;92-111. Disponible en: http://www. caunet.org/images/Libros/urofisioterapia.pdf.

3. Tyagi S, Thomas CA, Hayashi Y, Chancellor MB. The overactive bladder: Epidemiology and morbidity. Urol Clin North Am 2006;33(4):433-8.

4. Dávila $H$, Nieves L, Arocha J. Primer consenso venezolano en vejiga hiperactiva. Sociedad Venezolana de Urología. Caracas, 2005. Disponible en: http://www.soveuro.org.ve/especialistas/wp-content/ uploads/2015/02/libro_consensoConsensoVenezolanodeVejigaHiperactiva.pdf.

5. de Boer TA, Slieker-ten Hove MC, Burger CW, Vierhout ME. The prevalence and risk factors of overactive bladder symptoms and its relation to pelvic organ prolapse symptoms in a general female population. Int Urogynecol J 2011;22(5):569-75. 
6. Palma P, Dávila H. Uroginecología. Paulo P, Dávila $\mathrm{H}$. (Eds) Confederación Americana de Urología. Caracas, 2006:39-68.

7. Kalsi V, Apostolidis A, Gonzales G, Elneil S, Dasgupta $P$, Fowler CJ. Early effect on the overactive bladder symptoms following botulinum neurotoxin type A injections for detrusor overactivity. Eur Urol 2008;54(1):181-7.

8. Andersson KE, Appell R, Cardozo L, et al. Pharmacological treatment of urinary incontinence. En: Abrams $P$, Khoury S, Wein A (ed). Incontinence: 3rd International Consultation on Incontinence. Paris, France: Health Publications;2005. pp 809-54.

9. Chapple CR, Khullar V, Gabriel Z, Muston D, Bitoun CE, Weinstein D. The effects of antimuscarinic treatments in overactive bladder: an update of a systematic review and meta-analysis. Eur Urol 2008;54:543-62.

10. Starkman JS, Smith CP, Staskin DR. Surgical options for drug-refractory over active bladder patients. Rev Urol 2010;12(2-3):e97-e110.

11. Kessler TM, Bachmann LM, Minder C, Löhrer D, Umbehr $\mathrm{M}$, et al. Adverse event assessment of antimuscarinics for treating overactive bladder: a network meta-analytic approach. PLoS ONE 2011;6(2):e16718.

12. Hashim $H$, Abrams $P$. How should patients with an overactive bladder manipulate their fluid intake? BJU Int 2008;102(1):62-6.

13. Srikrishna S, Robinson D, Cardozo L, Vella M. Management of overactive bladder syndrome. Postgrad Med J 2007;83(981):481-6.

14. Mangera A,Chapple C. Uso de la toxina botulínica en el tratamiento de los trastornos del tracto urinario inferior. Arch Esp Urol 2010;63(10):829-41.

15. Giannantoni A, Mearini E, Del Zingaro M, Porena M. Six-yearfollow-up of botulinum toxin A intradetrusorial injections in patients with refractory neurogenic detrusor over activity: clinical and urodynamic results. Eur Urol 2009;55(3):705-11.

16. Del Popolo G, Filocamo MT, Li Marzi V, MacchiareIla A, Cecconi F, Lombardi G, Nicita G. Neurogenic detrusor over activity treated with English botulinum toxin a: 8-year experience of one single centre. Eur Urol 2008;53(5):1013-19.

17. Pannek J, Göcking K, Bersch U. Long-term effects of repeated intradetrusor botulinum neurotoxin $A$ injections on detrusor function in patients with neurogenic bladder dysfunction. BJU Int 2009;104(9):1246-50.

18. Apostolidis A, Jacques TS, Freeman A, Kalsi V, Popat R, Gonzales G, Datta SN, Ghazi-Noori S, Elneil $S$, Dasgupta P, Fowler CJ. Histological changes in the urothelium and suburothelium of human overactive bladder following intradetrusor injections of botulinum neurotoxin type $A$ for the treatment of neurogenic or idiopathic detrusor overactivity. Eur Urol 2008;53(6):1245-53.

19. Sahai A, Dowson C, Khan MS, Dasgupta P. Improvement in quality of life after botulinum toxin-A injections for idiopathic detrusor overactivity: results from a randomized double-blind placebo-controlled trial. BJU Int 2009;103(11):1509-15.

20. Shah S, Nitti VW. Defining efficacy in the treatment of overactive bladder syndrome. Rev Urol 2009;11(4):196202.

21. Coyne KS, Matza LS, Kopp Z, Abrams P. The validation of the patient perception of bladder condition (PPBC): a single-item global measure for patients with over active bladder. Eur Urol 2006;49(6):1079-86.

22. Martínez CB, Salinas SA, Giménez BJM, Donate MJ, Pastor NH, Virseda RJA. Calidad de vida en las pacientes con incontinencia urinaria. Actas Urol Esp 2008;32(2):202-10.

23. Patel AK, Patterson JM, Chapple CR. Botulinum toxin injections for neurogenic and idiopathic detrusor overactivity: a critical analysis of results. Eur Urol 2006;50(4):684-710.

24. Yoshida M, Masunaga K, Nagata, Yono M, Homma M. The forefront for novel therapeutic agents based on the pathophysiology of lower urinary tract dysfunction: pathophysiology and pharmacotherapy of overactive bladder. J Pharmacol Sci 2010;112(2):128-34. 